\title{
Optical Feeder links for High Throughput Satellites
}

\author{
Bernard Roy ${ }^{1}$, Sylvain Poulenard ${ }^{1}$, Svilen Dimitrov ${ }^{2}$, Ricardo Barrios $^{2}$, Dirk Giggenbach ${ }^{2}$, Arnaud Le \\ Kernec $^{3}$, Michel Sotom ${ }^{3}$ \\ 1-Airbus Defence and Space, Toulouse,France,Bernard.Roy@airbus.com,Sylvain.Poulenard@airbus.com \\ 2- DLR-IKN,Weßling, Germany,Svilen.Dimitrov@dlr.de, Ricardo.Barrios@dlr.de,Dirk.Giggenbach@dlr.de \\ 3- Thales Alenia Space, Toulouse, France, Arnaud.Le-Kernec@thalesaleniaspace.com, \\ Michel.Sotom@thalesaleniaspace.com
}

\begin{abstract}
Optical feeders for geostationary High Throughput Satellites (HTS) systems based on $1.55 \mu \mathrm{m}$ wavelength technology are expected to enable to transmit up to several terabits over one active link. A desirable option of transmission architecture is an optical feeder link transparent with respect to the user air interface. This can be implemented using either a digital or an analog modulation of the optical carrier. The digital option increases the optical bandwidth to be transmitted, however it benefits from error correcting codes, interleaving and framing which are efficient against atmospheric turbulence impairments. The analog option is more efficient concerning the optical bandwidth; however the atmospheric turbulence impairments can only be mitigated by a more complex optical ground terminal. Both analog and digital options could be feasible in the 2025-2030 time-frames but the digital option is more mature with respect to the atmosphere impairments mitigation techniques.
\end{abstract}

Index terms- high throughput satellite; laser communication; free-space optical communications;

\section{INTRODUCTION}

The next generation of HTS will play an important role in the fulfilment of the Digital Agenda objectives by offering both very high throughput and data rates. Among all technological options for the feeder links, we have considered in the research project BATS [1] (Broadband Access via integrated Terrestrial \& Satellite systems) an optical feeder link, as illustrated on Figure 1, which has the potential to become an alternative to or more probably the next step after conventional radio-frequency solutions, e.g. in $\mathrm{Q} / \mathrm{V}$ band [2], currently in development. This approach enables in theory the use of a single active optical link per satellite thanks to a very large available bandwidth (BW) and a high telescope gain. However, many challenges have to be addressed to consolidate the outline of an optical feeder link including the high level of availability required for feeder links (addressed in [3]), the transmission through atmospheric turbulences (addressed in [4]) and the choice of the modulation of the optical carrier.
The present paper deals with the analysis of the analog and digital modulations options on the optical feeder link, their impact on the feeder link architecture and the related mass and power consumption (PC) of the on-board hardware. Finally, a recommendation on the optical modulation is provided.

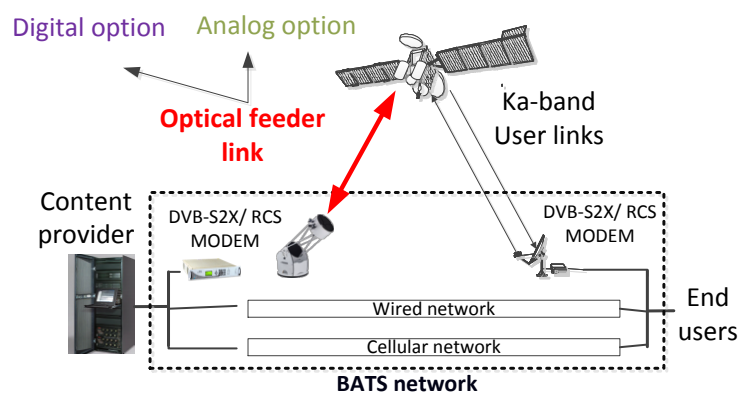

Figure 1: BATS with optical feeder

\section{USER LINK DESCRIPTION}

The proposed design for BATS is based on two geostationary co-located HTS covering EU27 plus Turkey, allowing more than $1 \mathrm{Tbps}$ in total. The capacity per satellite is $400 \mathrm{Gbps}$ in the forward (FWD) and 125 Gbps in the return links (RTN). The user beam layout is based on 302 small user beams of $0.21^{\circ}$ of beam width, 151 for each satellite, covering the East and West side of the coverage respectively.

User link operates in Ka-band, making use of the fixed satellite service exclusive and shared spectrum in both forward and return links. On the forward downlink, 1.45 $\mathrm{GHz}$ of spectrum is allocated per user spot beam, based on a regular 4-colour frequency reuse scheme. Assuming a 5\% roll-off factor, 3 carriers of 425 Msps can be accommodated in the forward link. On the return uplink, on average, $525 \mathrm{MHz}$ of spectrum is allocated per user spot beam, being also based on a regular 4-colour frequency reuse scheme.

\section{WHY AN OPTICAL FEEDER LINK AT $1.55 \mu \mathrm{m}$}

The interest of optical link at $1.55 \mu \mathrm{m}$ wavelength for the feeder of HTS is pushed by several factors. It can use 
optical fibre terrestrial technology like dense wavelength division multiplexing technique for the multiplexing of channels and high power Erbium Doped Fiber Amplifier (EDFA) booster amplifiers to provide very high throughput. In this way, it is expected to drastically reduce the number of gateway stations required on the feeder link when compared to classical Radio-Frequency (RF) feeder. Due to the very narrow beam-width, the link is immune to interferences. The active optical feeder being in relation with all user beams has knowledge of all interferers and can ease the implementation of interference management techniques to further improve the system throughput. It should be noted that for $\mathrm{Q} / \mathrm{V}$ feeder the performance of such techniques is constrained by the fact that each gateway handles a limited number of user beams. The latter feature also presents the advantage to avoid any frequency coordination.

\section{OPTICAL FEEDER LINK MODULATION}

The Ka band user signals use the DVB-S2X (forward) and DVB-RCS2 (return) communication standards in the BATS system. In order to avoid the implementation of the modulation/demodulation of these standards on-board the satellite (which increases the on-board hardware and disallows flexibility), a desirable option is an optical feeder link transparent with respect to the user air interface.

We have the choice between two possible types of modulation to implement the transparent optical feeder links: either modulation with a digital technique, or with an analog technique. While using partly common equipment these two options impact differently the onboard and on-ground optical hardware, the interface with the RF user payload and the operation of the laser link. Both options have been comprehensively analysed in the framework of BATS.

The analog option uses RF over Fibre techniques used on ground to transport RF signals efficiently: the wellknown advantages of optical fiber as a transmission medium such as low loss, light weight, large bandwidth characteristics, small size and low cable cost make it the ideal and most flexible solution for efficiently transporting radio signals to remotely located antenna sites.

The digital option is based on the well-known terrestrial fiber communication technique which is able to transport several terabit/s on optical fiber backbones and is currently implemented worldwide. It allows using well mastered mitigation techniques (and the related hardware) against random errors. However the digitalisation of the data to be transmitted increases the required bandwidth.

In addition to specific architectures, both options have to deal with the atmospheric channel impairments (in terms of random errors and fading), using common or different techniques.

\section{ATMOSPHERIC TURBULENCE MITIGATION}

The optical feeder analyzed hereafter aims at transferring very high data rate signals through the atmospheric channel. The atmospheric channel introduces perturbations which have to be mitigated in both downlink and uplink.

In the downlink, the signal received by the telescope of the On-Ground station (OGS) is impacted by intensity fluctuations (scintillation) and phase distortion. While the phase perturbations of the received waveform modify the interfering pattern at the focal point of the telescope (random displacement of the focal point spot or spread in the form of multiple spots), the scintillation phenomenon creates instantaneous variation of the power received by the telescope.

In the uplink, the signal received by the telescope of the satellite is impacted by the on axis amplitude scintillation and the beam wandering. The later can be seen as the result of a random angular tilt at transmit level. The resulting displacement of the centre of the spot at satellite receiver level induces also a fluctuation of the received irradiance. The high order of the phase distortion in the atmospheric layer results in broadening of the beam. The phase variation of the optical wave - across the telescope aperture - at the satellite plane is negligible, as the beam spot is much larger than receiver diameter. Ultimately, this translates into minimum fibre coupling loss at the GEO satellite optical receiver chain.

A consequence of the asymmetry between the uplink and downlink channels is that the mitigation methods are different. One remark is that the opto-mechanical mitigation methods are all implemented at OGS level and do not complicate the on board terminal. This is not the case for the communication mitigation methods like Forward Error Code (FEC) which may imply the need for on-board signal processor. A second remark is the tracking function of the OGS is critical, because it impacts the receive performances of the downlink (through the tip-tilt correction) and the pointing performances of the uplink and therefore the beam wandering.

Downlink opto-mechanical mitigation techniques

Due to the high data throughput, a highly sensitive detection process must be used. Therefore, it is assumed that the receiver is composed of a pre-receiver singlemode EDFA followed by a PIN diode. The link budget must also be maximized, involving the use of large aperture receive telescope in the downlink. This reduces the amplitude scintillation, but in the same time decreases the coherence of the phase of the signal received over the aperture. The consequence is a spread of the signal spot in front of the core of the EDFA single mode fiber located at the focal point of the telescope and a dramatic decrease of the power coupled into the fiber. To compensate for the high orders of the wave front errors and to concentrate the energy in the plane of the single mode fiber core, Adaptive Optics (AO) system can be implemented (no detailed analysis of the AO has been performed in the frame of BATS).

\section{Uplink opto-mechanical mitigation techniques}

The multi-aperture transmitter (Tx), also called transmitter diversity, allows reducing the uplink scintillation thanks to the combination of mutually incoherent beams. To efficiently implement this technique, it must be ensured that the laser signals received by the on-board telescope are spectrally incoherent. At the $1.55 \mu \mathrm{m}$ wavelength, considering the 
spectral linewidth of the laser diode source, this requirement can only be achieved by spectrally separating the modulated optical signals, avoiding overlaps but inducing an increase of the used wavelength bandwidth (e.g. for a $\mathrm{Tx}$ diversity of 2, the optical spectrum bandwidth needs to be doubled).

\section{Fading mitigation techniques}

The fading mitigation techniques are suitable for the digital option as they need to have the data in digital format. Some error correcting codes, like low-density parity-check, are able to correct random errors. To deal with fading events, one can either use interleavers which allow spreading burst errors induced by fading into small length burst which can be corrected by the random error correcting codes or erasure correcting codes which are proposed in [4].

\section{OPTICAL LINK BUDGET PER CHANNEL}

To ensure the requested user capacity for one satellite, a carrier to noise ratio greater than $17 \mathrm{~dB}$ in the forward and $18 \mathrm{~dB}$ in the return is required on the optical feeder.

The on-board terminals are sized to support the very high throughput, with a telescope diameter of $250 \mathrm{~mm}$ and up to $10 \mathrm{~W}$ per optical channel. The on-ground terminals are sized with a telescope diameter of $500 \mathrm{~mm}$ on the receive side and $200 \mathrm{~mm}$ on the transmit side (to minimize the beam wandering effect) and $50 \mathrm{~W}$ per optical channel. For each optical link, a redundant optical terminal (named passive in Table 2) is implemented on board the satellite to minimize the time interruption during the handover.

In both analog and digital option, the receiver is based on a pre-receiver EDFA followed by a PIN detector which provides the best sensitivity for direct detection.

The compensation of the atmospheric turbulence induced perturbations is achieved in the on-ground terminal thanks to AO system for the downlink (all options) and multiple transmitters for the uplink (uncoded digital and analog options).

The total available optical bandwidth is $60 \mathrm{~nm}$ by concatenating the optical C- and L-bands. The channel wavelength multiplexing is based on the ITU grid [5]. The link budgets hypotheses are displayed in Table 1 .

\begin{tabular}{|l|r|r|r|}
\hline Parameter & Uplink & $\begin{array}{c}\text { Downlink } \\
\text { digital }\end{array}$ & $\begin{array}{c}\text { Downlink } \\
\text { Analog }\end{array}$ \\
\hline Tx diameter [cm] & 20.0 & 25.0 & 25.0 \\
\hline Tx power [W] & 50.0 & 1.0 & 10 \\
\hline Tx power [dBm] & 47.0 & 30.0 & 40 \\
\hline Tx antenna gain [dB] & 112.2 & 114.1 & 114.1 \\
\hline Tx optical loss [dB] & -3.0 & -3.0 & -3.0 \\
\hline Tx pointing loss [dB] & -1.7 & -0.7 & -0.7 \\
\hline Free-space loss [dB] & -290.4 & -290.4 & -290.4 \\
\hline Absorption loss [dB] & -1.0 & -1.0 & -1.0 \\
\hline Cloud margin [dB] & -3.0 & -3.0 & -3.0 \\
\hline Scintillation margin [dB] & $-\left(^{*}\right)$ & -1.9 & -1.9 \\
\hline Rx diameter [cm] & 25.0 & 50.0 & 50.0 \\
\hline Rx antenna gain [dB] & 114.1 & 120.1 & 120.1 \\
\hline Rx optical loss [dB] & -3.0 & -3.0 & -3.0 \\
\hline Total Rx power [dBm] & $\mathbf{- 2 8 . 8}$ & $\mathbf{- 3 8 . 8}$ & $\mathbf{- 2 8 . 8}$ \\
\hline
\end{tabular}

Table 1 : Uplink and downlinks budgets

(*)the uplink scintillation is elaborated from the time series
For the downlink, the scintillation margin includes the remainder of the intensity scintillation averaged by the telescope aperture and the phase perturbation corrected by AO systems. For the uplink, the intensity scintillation at receiver level is simulated through an atmospheric turbulence model derived from [11] resulting in time series illustrated on Figure 2. For the digital option, the resulting power fluctuation is included in the NRZ-OOK model as an instantaneous optical channel gain to derive the average bit-error rate in the fading channel. For the analog option, uplink scintillation is mitigated by transmitter diversity.

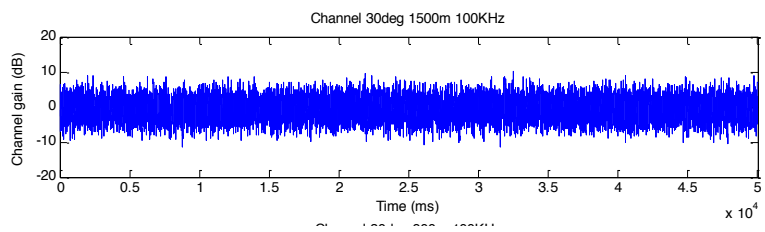

Figure 2: Scintillation time series

\section{DIGITAL MODULATION ARCHITECTURE}

The digital modulation is based on NRZ-OOK and is generally performed by intensity modulation of an optical carrier by an Mach-Zehnder modulator [4]. Depending on the link budget, FEC coding must be implemented to counterbalance the fading channel (fade duration is in the order of tens of milliseconds). It is proposed to use erasure packet level code in one option and no code in a second option.

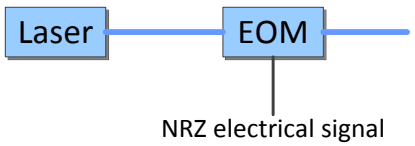

Figure 3: Optical NRZ-OOK modulation

\section{Coded digital modulation}

In the forward direction, after DVB-S2X modulation of the user signals, two optical links of 75 data streams carrying $1.45 \mathrm{GHz}$ each are transmitted. The optical grid is $50 \mathrm{GHz}(0.4 \mathrm{~nm})$. Digitalization of real and imaginary components sampled at the Nyquist rate, uses a 6 bit analog to digital converter (ADC) per dimension, ensuring a signal-to-distortion ratio of up to $36 \mathrm{~dB}$. After separation of the real and imaginary components, the bit stream is multiplexed and fed to the FEC encoder. The erasure code is composed of an inner code (Bose - Ray Chaudhuri - Hocquenghem) concatenated with a sparsegraph-based erasure correcting LDPC (Low Density Parity Check) code. The encoded bit stream is then passed to the optical section including two stages of wavelength multiplexers (low then high power) and booster amplifiers before transmitting through the telescope. After reception and demultiplexing by the onboard optical terminal, the 75 data streams are decoded with a digital on-board processor requiring a memory block of estimated 12 GBytes (75 FPGAs with memory of at least 162.5 Mbytes each), in order to counter the long fading events. Finally, the data stream is converted through a digital to analog converter back to baseband DVB-S2 and up-converted to Ka band at the user payload interface. 
In the return link, no FEC coding is needed, thanks to the implementation of large aperture and $\mathrm{AO}$ systems in the OGS. The signal received at the user payload interface in DVB-RCS2 format is sampled at the Nyquist rate then digitalised using a 6 bits ADC per dimension. Due to the favourable link budget, no FEC coding is implemented in the return link. The two optical links of 75 data streams (each are passed to the optical payload including two stages of wavelength multiplexers (low then high power) and booster amplifiers before transmitting through the telescope. The optical grid is $25 \mathrm{GHz}(0.2 \mathrm{~nm})$. After reception and demultiplexing by the on ground optical terminal, the data stream is converted through a digitalto-analog converter back to baseband DVB-RCS2 then up-converted to L band. As depicted in Figure 4, two optical links with wavelength re-use are needed to carry the total throughput. They are located at a distance greater than $2 \mathrm{~km}$ to allow separation of the two beams at the on-board receiver level.

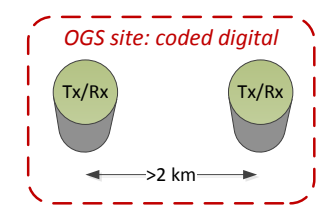

Figure 4: Ground station for digital coded option

\section{Uncoded digital modulation}

In the forward direction, considering the static link budget and the uplink scintillation time series, it is only possible to close the link budget in the uncoded option by considering a 2 transmitters (Tx) diversity scheme for scintillation mitigation and a transmitted power increased by the same amount (each Tx transmit $50 \mathrm{~W}$ power which provides $3 \mathrm{~dB}$ additional margin). The optical grid is 100 $\mathrm{GHz}(0.8 \mathrm{~nm})$. Wavelength reuse is necessary to cope with the overall available optical bandwidth; therefore the bandwidth is spread over three optical links of 50 data streams. Three clusters separated by $2 \mathrm{~km}$ each and including each two transmitters and one receiver (Rx) share the overall bandwidth. The transmission scheme implements the same function as the coded option except the FEC encoding for the forward link. As depicted in Figure 5, three optical links are needed to carry the total throughput.

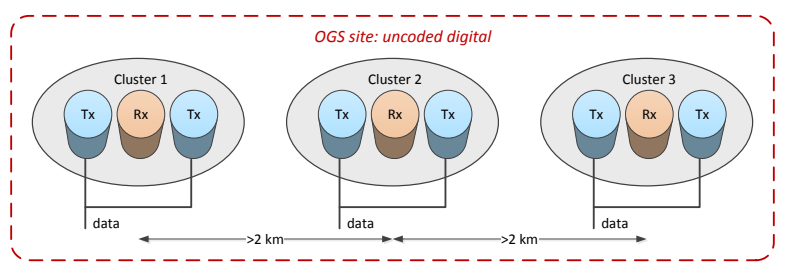

Figure 5: Ground station for digital un-coded option

The uncoded digital modulation option is attractive because it reduces the size of the on-board processor; however this is at the cost of increased complexity for the ground station and on board terminals due to the need of transmitter diversity for uplink scintillation mitigation.

\section{ANALOG MODULATION}

Analog modulation is achieved by modulating a continuous wave optical carrier through an electro-optic modulator.

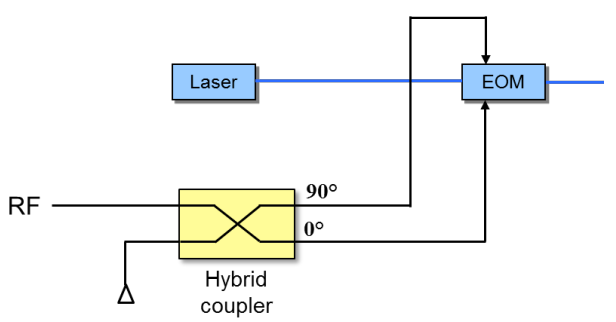

Figure 6: Single sideband modulation

Among the possible modulation the Single Side Band (SSB) has been selected, because it offers the advantage of reducing the occupied optical bandwidth by a factor of 2 compared to Double Side Band (DSB) modulation. Prior modulating the optical carrier, the RF signal must be frequency converted to an Intermediate Frequency (IF) ( $\mathrm{S}, \mathrm{C}, \mathrm{Ku}$ or $\mathrm{Ka}$ band). High IF simplifies the frequency up or down-conversion at the $\mathrm{Ka}$ band user payload interface but will exhibit poor spectrum efficiency in the optical channel. In the opposite, low IF increases drastically the up and down converters complexity, but it makes a more efficient use of the optical bandwidth.

Several configurations of IF and optical spectrum allocations options have been analysed considering the available overall optical bandwidth and the link budget.

\section{Forward link}

Considering the static link budget and the uplink scintillation time series, the link budget can only be closed, if a four Tx diversity scheme is implemented and the transmitted power is increased by the same amount (each Tx transmit $50 \mathrm{~W}$ power which provides $6 \mathrm{~dB}$ additional margin). The resulting scheme is a SSB modulation with C-band IF and $25 \mathrm{GHz}$ grid spacing with one $1.5 \mathrm{GHz}$ user beam per optical channel. With this scheme, four times 150 channels of $0.2 \mathrm{~nm}$ are spread over a three clusters configuration ( 3 clusters of $50 \lambda \times 3$ x $450 \mathrm{MHz}$ ).

\section{Return link}

The scheme is a SSB modulation with C-band IF and 25 $\mathrm{GHz}$ grid spacing with two beam spectra 2 x $(37 \times 12$ $\mathrm{MHz}$ ) per optical channel. The Rx 75 channels are spread over the three clusters of the on ground station (3 clusters of $25 \lambda \times 2 \times(37 \times 12 \mathrm{MHz}))$.

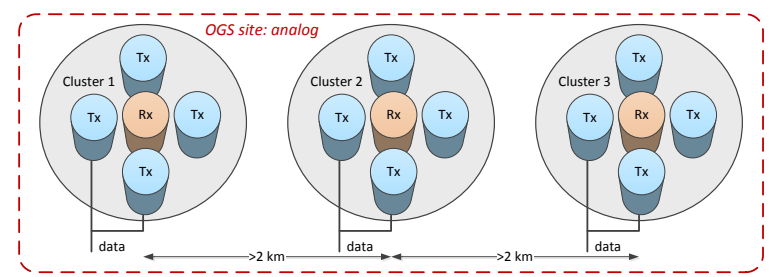

Figure 7: Ground station for analog option

Without any consideration related to the mitigation of propagation issues, the analog option seems at first very promising, in particular because no digital processor on- 
board is required, and the spectral occupancy is fully compliant with available optical bandwidth. However, the optical wavelength reuse for fading mitigation imposes severe constraints on ground stations dramatically increasing their complexity and imposes to duplicate the terminals on-board, increasing mass, power consumption and complexity of the payload. The cost of bandwidth expansion to mitigate fading is the increase of the mass and power consumption on-board and the increase of the terminal and ground stations complexity.

\section{TECHNOLOGICAL CHALLENGES}

Part of the components needed for the optical space terminal is already space qualified. This is the case of the structure (using Silicon Carbide technology which is an ideal material for building ultra-stable telescopes and optical benches), the thermal parts (like miniaturized space qualified flat loop heat pipe [8]), and the pointing mechanisms. The acquisition and tracking sensor the concept of which has been validated at $0.8 \mu \mathrm{m}$ [7] shall be developed at $1.55 \mu \mathrm{m}$ and qualified to radiations.

The optical communication chain relies on the $1.5 \mu \mathrm{m}$ technology, initially developed for terrestrial networks and several components have a high Technical Readiness Level (TRL) but need to be qualified for a space environment. The key components to be specifically developed and qualified for the space terminal include high power amplifiers, high power multiplexers and digital processors.

High power booster amplifiers are a key issue for feeder links due to the high level of optical power requested. Current terrestrial amplifier must be significantly improved in terms of wall plug efficiency and robustness to radiation. A wall plug efficiency of $20 \%$ could be targeted in the 2025 timeline for the Erbium doped fiber thanks to the use of in-band pumping.

The multiplex of tens of high power optical channels will handle a thermal load of several hundreds of Watts. The multiplexer must exhibit very low insertion loss and ensure an efficient coupling towards the telescope. Bulk optic based on volume Bragg grating has already been proposed to combine very high power laser beam and may be a suitable candidate for feeder link applications.

The digital processor (for digital option only) needs high capacity FPGAs expected to be available in the 2025 timeline.
Another technological challenge is related to the power handling capability of the telescope of the OGS. Some LIDAR [12] which can emit laser pulses generating heat of about $100 \mathrm{~W}$ have been space qualified but the behaviour of similar optics in the permanent presence of more than hundreds of watt must be qualified.

\section{ANALOG AND DIGITAL OPTIONS TRADE-OFF}

The trade-off between the analog and the two digital options has been performed considering the mass and power consumption budgets (including in these budgets the interface with the user payload such as the up and downconverters and necessary RF filters) as well as the bandwidth occupancy (considering the Tx diversity scheme) and the TRL level.

The budgets are based on hardware expected with performances available for flight in the 2025 timeline.

The first remark is that considering the technological challenges, the optical feeder for HTS can only be envisaged in the 2025-2030 timeline, either for the digital or the analog options. The main differences between the digital and analog options are summarized in Table 2.

The main drawback of the digital option is that it expands the user signal bandwidth by a factor greater than 10 (corresponding to the DAC/ADC resolution) which requests very large optical bandwidth, exceeding in our case the capability of the C plus L optical bands, resulting in multiple clusters. The coded digital option offers error correction code technique which allows coping with the atmospheric turbulence impairments (in particular in the uplink) without complex on ground technique like transmitter diversity. The major question is the timeline of the feasibility of the on board processor implementing this FEC scheme.

The analog option makes an efficient use of the optical bandwidth due to the fact that the RF user signal is modulating directly the laser. However, without Tx diversity technique in the uplink, it is not possible to close the link budget for the analog option. The implementation of the Tx diversity technique involves an increase by a factor of $\sim 3$ of the optical bandwidth (as well as by a factor of 4 for the optical power per wavelength), which, in some way, annuls the intrinsic bandwidth advantage of the analog option which is also shown to require multiple clusters.

\begin{tabular}{|c|c|c|c|c|c|c|c|c|c|c|}
\hline & \multicolumn{5}{|c|}{ Geostationnary satellite } & \multicolumn{5}{|c|}{ Optical ground station } \\
\hline & \multirow{2}{*}{$\begin{array}{l}\text { Nb on } \\
\text { board } \\
\text { term. }\end{array}$} & \multicolumn{2}{|c|}{ Optical bandwidth } & \multirow{2}{*}{$\begin{array}{c}\text { Tot. } \\
\text { mass } \\
(\mathrm{kg})\end{array}$} & \multirow{2}{*}{$\begin{array}{l}\text { Tot. } \\
\text { PC } \\
\text { (W) }\end{array}$} & \multicolumn{3}{|c|}{ Atmospheric mitigation } & \multirow{2}{*}{$\begin{array}{l}\text { Architec } \\
\text {-ture }\end{array}$} & \multirow{2}{*}{$\begin{array}{c}\text { Tot. } \\
\text { PC } \\
(\mathbf{k W})\end{array}$} \\
\hline & & $\begin{array}{l}\text { FWD/RTN } \\
\text { bandwidth } \\
(\mathrm{nm})\end{array}$ & $\begin{array}{l}\text { Tot. } \\
\text { BW } \\
\text { (nm) }\end{array}$ & & & $\begin{array}{l}\text { FWD } \\
\text {-FEC }\end{array}$ & $\begin{array}{l}\text { Downlink } \\
\text { feature }\end{array}$ & $\begin{array}{l}\text { Uplink } \\
\text { feature }\end{array}$ & & \\
\hline $\begin{array}{l}\text { Digital } \\
\text { FWD- } \\
\text { coded }\end{array}$ & $\begin{array}{l}2 \text { active } \\
+2 \\
\text { passive }\end{array}$ & $\begin{array}{l}\text { FWD: } 2 \times 30 \\
\text { RTN: } 2 \times 15\end{array}$ & 90 & 771 & 5987 & yes & $\begin{array}{l}\text { AO } \\
\text { Large } \mathrm{SA}^{2}\end{array}$ & no $\mathrm{MA}^{1}$ & $\begin{array}{l}2 \text { clusters } \\
(1 \mathrm{Tx}, 1 \\
\mathrm{Rx})\end{array}$ & $\begin{array}{c}2 \mathrm{x} \\
3.75\end{array}$ \\
\hline $\begin{array}{l}\text { Digital } \\
\text { FWD- } \\
\text { uncoded }\end{array}$ & $\begin{array}{l}3 \text { active } \\
+3 \\
\text { passive }\end{array}$ & $\begin{array}{l}\text { FWD: } 3 \times(20+20) \\
\text { RTN: } 3 \times 10\end{array}$ & 150 & 867 & 6093 & no & $\begin{array}{l}\text { AO } \\
\text { Large SA }\end{array}$ & $\begin{array}{l}2 \mathrm{Tx} \\
\mathrm{MA}\end{array}$ & $\begin{array}{l}3 \text { clusters } \\
(2 \mathrm{Tx}, 1 \\
\mathrm{Rx})\end{array}$ & $3 \times 5$ \\
\hline $\begin{array}{l}\text { Analog } \\
\text { SSB }\end{array}$ & $\begin{array}{l}3 \text { active } \\
+3 \\
\text { passive }\end{array}$ & $\begin{array}{l}\text { FWD: } 3 \times(10+10 \\
+10+10) \\
\text { RTN: } 3 \times 5\end{array}$ & 135 & 823 & 9372 & N/A & $\begin{array}{l}\text { AO } \\
\text { Large SA }\end{array}$ & $\begin{array}{l}4 \mathrm{Tx} \\
\mathrm{MA}\end{array}$ & $\begin{array}{l}3 \text { clusters } \\
(4 \mathrm{Tx}, 1 \\
\mathrm{Rx})\end{array}$ & $\begin{array}{l}3 \mathrm{x} \\
10\end{array}$ \\
\hline
\end{tabular}

1. $\mathrm{MA}=$ Multiple Aperture (for Tx diversity) / 2. $\mathrm{SA}=$ Single Aperture (Receive telescope)

Table 2 : Main features of analog and digital optical feeder options 
Although the transmitter diversity technique has been successfully implemented already in several experiments [9], [10], its applicability to very high throughput feeder links is a point of concern which needs to be specifically addressed.

The uncoded digital option is in between the two previous options as the link budget can be closed with moderate 2Tx diversity. This option allows to reduce the complexity of the on board processor.

Pre-adaptive optics for the uplink has been analyzed in the frame of BATS, but it has not been taken into account as mitigation technique. The level of maturity of this technique is currently low. Its implementation could improve both options capability to deal with the atmospheric turbulence impairments. Another potential improvement for the digital option could be to increase the spectral efficiency, e.g with D-QPSK modulation.

BATS analysis has shown the interest of an optical feeder link for both analog and digital options. Assuming dedicated efforts, the TRL level may be compatible with an operational system in the 2025-2030 timeline. However, despite the criticality of the on-board processor, the digital option appears to be more mature considering the implementation of atmosphere impairments mitigation techniques.

\section{CONCLUSION AND PERSPECTIVES}

The study of the optical option for the feeder link in the frame of the BATS project has shown that an optical feeder link for high throughput feeder may be feasible. Two options have been analyzed on the basis of a transparent feeder link with respect to the user signal: analog and digital options. The performance of both options has been analyzed considering the atmospheric turbulence impairments. It has been shown that with both options it was possible to close the link budget by using the relevant atmospheric perturbation mitigation methods implemented in the optical ground station:

- For the downlink: use of large receive aperture and adaptive optics to maximize the collected flux and signal injected into the EDFA pre-receiver (best receive sensitivity direct detection receiver)

- For the uplink: use of error correction codes (not relevant for analog option) or transmitter diversity (for both options)

Considering 2025-2030 time frame, the digital option has been preferred considering the error correcting code capability on the forward link that limits the optical bandwidth and power required the on-ground station (no code necessary on the downlink). The implementation of some mitigation methods involves several constraints on which we need to work out in the coming period to have the technology available in the 2025-2030 time-frame:

- Signal processor

- Control of transmitter diversity (telescope pointing, data synchronization)

- $\quad$ Adaptive Optics (control bandwidth).

The very high throughput is also very demanding in terms of laser source on board: drastic technology improvement is required to increase the wall plug efficiency of power booster in the range of $20 \%$. Finally, several components (including the acquisition and tracking sensor) must be developed and qualified with the objective to be available within 2025-2030 time frame.

\section{ACKNOWLEDGEMENTS}

This work has been supported by the BATS research project which is funded by the European Commission Seventh Framework Programme (FP7) under contract n.317533. The authors of this paper would like to acknowledge the European Commission FP7 Programme and the members of the BATS Consortium.

\section{REFERENCES}

[1] BATS FP7 Project public website, http://www.batsproject.eu

[2] J. Pérez-Trufero, B. Evans, A. Kyrgiazos, M. Dervin, B. Garnier, C. Baudoin, "High Throughput Satellite System with Q/V-band gateways and its integration with terrestrial boradband communication networks", $32^{\text {nd }}$ AIAA International Communications Satellite Systems Conference, San Diego, 2014.

[3] S. Poulenard, M. Crosnier, and A. Rissons, "Ground segment design for broadband geostationary satellite with optical feeder link," Journal of Optical Communication and Networking, 2015.

[4] Svilen Dimitrov, Balazs Matuz, Gianluigi Liva, Ricardo Barrios, Ramon Mata-Calvo, Dirk Giggenbach, "Digital Modulation and Coding for Satellite Optical Feeder Links", $20147^{\text {th }}$ ASMS $/ 13^{\text {th }}$ SPSC

[5] International Telecommunication Union (ITU); Recommendation ITUR G.694.1, "Spectral Grids for WDM Applications: DWDM Frequency Grid,” 2012.

[6] G. Liva, E. Paolini, and M. Chiani, "Bounds on the Error Probability of Block Codes over the q-Ary Erasure Channel," IEEE Trans. Commun., vol. 61, no. 2, pp. 2156-2165, Jun. 2013.

[7] L. Vaillon, G. Planche, V. Chorvalli, L. Le Hors, "Optical Communications between an Aircraft and a GEO Relay Satellite: Design \& Flight Results of the LOLA Demonstrator." Proc. ICSO Conference 2008, Toulouse

[8] A. E. Marini, "ATLID: The Technology Development Programme for ESA's Satellite-borne Atmospheric Lidar", ESA bulletin 95, August 1998

[9] D. M. Boroson, B. S. Robinson, D. V. Murphy, D. A. Burianek, F. Khatri, J. M. Kovalik, Z. Sodnik, and D. M. Cornwell, "Overview and results of the lunard laser communication demonstration," Proc. Free-Space Laser Communication Technologies, 2014.

[10] R. Mata Calvo, P. Becker, D. Giggenbach, F. Moll, M. Schwarzer, M. Hinz, Z. Sodnik "Transmitter diversity verification on ARTEMIS geostationary satellite" Proc. SPIE Conference Free-Space Laser Communication and Atmospheric Propagation 2014

[11] L. C. Andrews and R. L. Philips, Laser Beam Propagation through Random Media, 2nd ed. Belligham: SPIE Press, 2005

[12] AEOLUS payload, [available on-line]:

http://www.esa.int/Our Activities/Observing the Earth/T he Living Planet Programme/Earth Explorers/ADM$\underline{\text { Aeolus/Payload }}$ 